\title{
Visualization of a Pseudospark-Sourced Electron Beam
}

\author{
David Bowes, Helen Yin, Wenlong He, Kevin Ronald, Alan D. R. Phelps, Defeng Chen, Peng Zhang, \\ Xiaodong Chen, Daohui Li, and Adrian W. Cross
}

\begin{abstract}
A pseudospark (PS)-sourced electron beam of 3-mm diameter has been experimentally investigated. Emission of X-rays was detected during a PS discharge and clear X-ray images were formed using the PS-sourced electron beam impacting on a 0.1-mm-thick molybdenum target at an applied voltage of $46 \mathrm{kV}$. Using a phosphor-coated scintillator, the beam's cross-sectional profile and surrounding ion channel were also observed. These results confirm the presence of an electron beam.
\end{abstract}

\section{Index Terms-Pseudospark, x-ray generation.}

$\mathbf{T}$ HE pseudospark (PS) discharge is a relatively new class of gas discharge, having only been discovered in the late 1970s. It is a self-sustained, transient, low pressure (typically 6.5-65 Pa) gas discharge in a hollow cathode/planar anode configuration, whose behavior varies with geometry and external circuit configuration. Additionally, an electron beam is formed during the breakdown process, which requires no guiding magnetic field due to the presence of an ion channel [1], [2]. The PS discharges have gained considerable attention during the last 30 years particularly with regard to their breakdown characteristics and the underlying plasma physics responsible for high current emission and potential applications in various fields. For example, they have been shown to act as a source of soft X-rays by means of X-ray fluorescence [3].

A four-gap, PS discharge structure with anode and cathode apertures of 3-mm diameter was constructed. The hollow cathode cavity was made of stainless steel, with a length of $50 \mathrm{~mm}$ and inner diameter of $50 \mathrm{~mm}$. These dimensions were based on the requirements of the hollow cathode effect [4]. An external energy storage capacitor of $428 \mathrm{pF}$ was placed

Manuscript received November 4, 2013; revised December 30, 2013; accepted January 10, 2014. This work was supported by the Engineering and Physical Sciences Research Council.

D. Bowes, H. Yin, W. He, K. Ronald, A. D. R. Phelps, and A. W. Cross are with the Department of Physics, SUPA, University of Strathclyde, Glasgow G4 0NG, U.K. (e-mail: david.bowes@ strath.ac.uk; h.yin@strath.ac.uk; w.he@ strath.ac.uk; k.ronald@strath.ac.uk; a.d.r.phelps@strath.ac.uk; a.w.cross@ strath.ac.uk).

D. Chen and P. Zhang are with the Computed Tomography Laboratory, School of Mathematical Sciences, Capital Normal University, Beijing 100048, China (e-mail: tianrolin@163.com; pzhang@mail.cnu.edu.cn).

$\mathrm{X}$. Chen and $\mathrm{D}$. Li are with the Department of Electronic Engineering, Queen Mary University of London, London E1 4NS, U.K. (e-mail: xiaodong.chen@elec.qmul.ac.uk; daohui.li@elec.qmul.ac.uk).

Color versions of one or more of the figures in this paper are available online at http://ieeexplore.ieee.org.

Digital Object Identifier 10.1109/TPS.2014.2300691 across the cathode and anode in the discharging circuit while a mechanical rotary pump evacuated the experimental system from the anode end through a vacuum valve. The working gas (argon) entered the chamber through a fine-controlled needle valve at the anode side, with pressure measured at the anode via a Baratron-type pressure gauge. The experimental setup and diagnostics are similar to [5].

A cross-sectional image of a PS -sourced, axially symmetric electron beam [Fig. 1(a)] was captured via the use of a long-exposure digital camera together with a scintillator disc. The scintillator disc consisted of $1-\mu \mathrm{m}$-thick copper foil coated in a fine layer of phosphorous powder and was situated in a drift tube downstream of the PS anode. The image shows a $3 \pm 0.3 \mathrm{~mm}$-diameter PS electron beam that has been captured. The central beam spot can clearly be seen, along with a surrounding halo, circled in Fig. 1(a). This ring of light forms as plasma electrons, generated from the ionization of background gas by the beam front, are expelled toward the edge of the drift tube by subsequent beam electrons so that an ion channel is formed for further beam propagation. The presence of this ring in the captured image shows that both the electron beam and the ion channel are present during the PS beam propagation.

While this is one method of indicating the presence of an electron beam, another is to detect the presence of X-rays when impacting the electrons on a suitable target. To this end, the scintillator was removed and a 0.1-mm-thick molybdenum target was placed immediately after the PS anode. The molybdenum target was also serving as a witness plate for the PS electron beam. An X-ray photodetector was placed 52 $\mathrm{mm}$ from the anode. A microsized object made of two crossed metal wires of $100 \mu \mathrm{m}$ in diameter was attached as a test object for X-ray imaging.

The PS was configured for operation in a free-running, as opposed to triggered mode. Critical breakdown pressure was noted after increasing gas pressure until breakdown occurs at a fixed applied voltage. By adjusting the needle valve while pumping, it was possible to maintain this pressure before applying an increasing voltage until breakdown occurred. In this way, repetitive breakdown was achieved with a repetition frequency of $1 \mathrm{~Hz}$. The X-ray detector was then able to acquire the image data instantaneously with each successive shot. By adjusting the gas pressure, X-ray images at a different discharge voltage, i.e., at different beam energy, were recorded. 

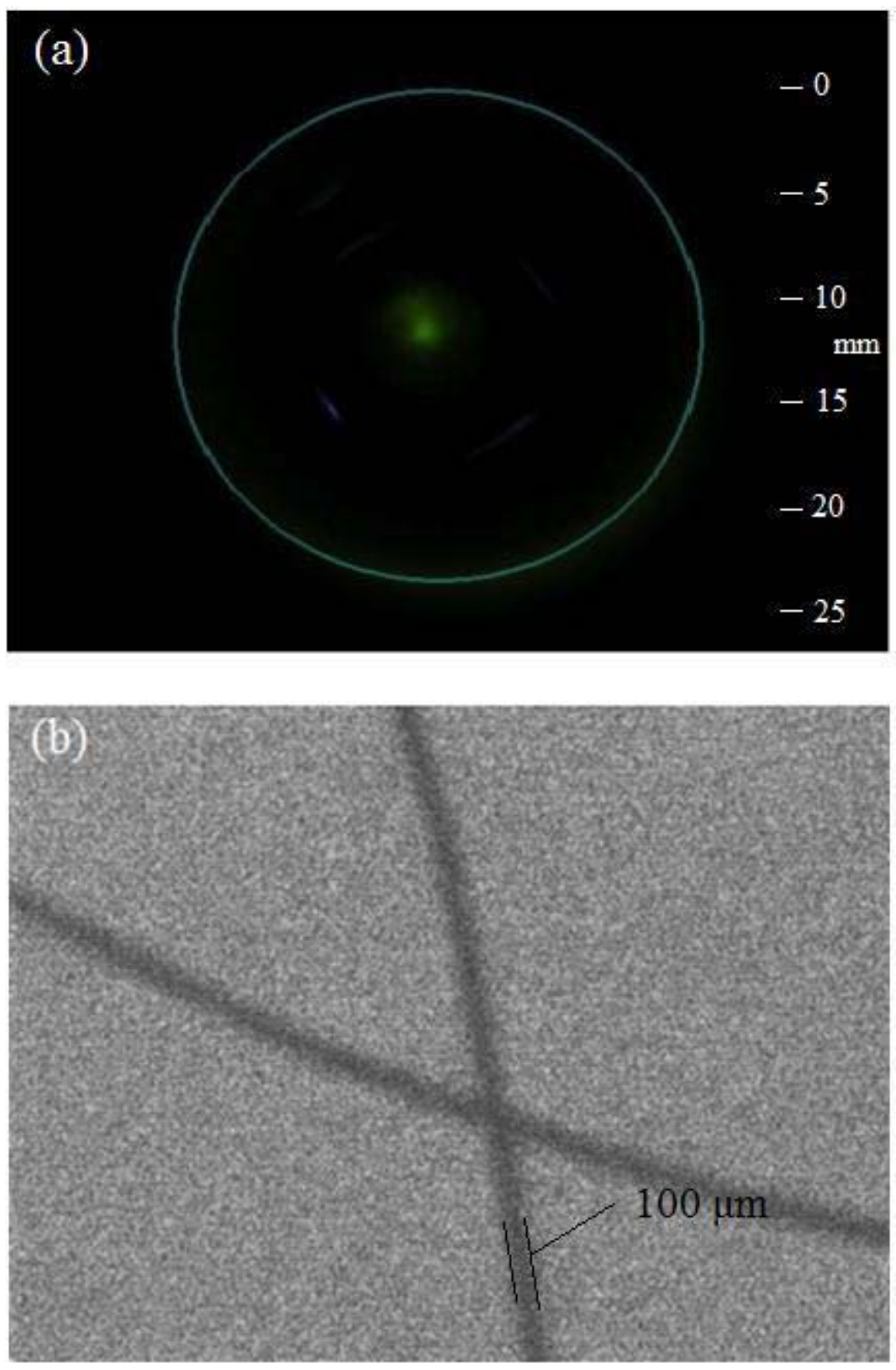

Fig. 1. (a) Cross-sectional image of $3 \mathrm{~mm}$-diameter pseudospark electron beam captured by means of a scintillator, and (b) image captured via the use of an X-ray photodetector.

Mean X-ray photon energies were calculated to be in the region of $19.8 \mathrm{keV}$. The integration time of the chargecoupled device detector was selected to be $800 \mathrm{~ms}$, with obtained images being captured from a single discharge pulse. $\mathrm{X}$-ray images of the crossed metal wires were recorded at voltages between 22 and $46 \mathrm{kV}$ with the clearest image [Fig. 1(b)] obtained at an applied voltage of $46 \mathrm{kV}$, corresponding to the production of a $300 \mathrm{~A}, 34-\mathrm{kV}$ PS-beam. The reduced clarity of the obtained images at lower voltages effectively set a threshold voltage for the effective capture of X-ray images from PS-sourced beams. In this way, the presence of the electron beam and its suitability as a small- spot X-ray imaging source were confirmed.

\section{REFERENCES}

[1] H. Yin, A. W. Cross, A. D. R. Phelps, D. Zhu, W. Hem, and K. Ronald, "Propagation and post-acceleration of a pseudospark-sourced electron beam," J. Appl. Phys., vol. 91, no. 8, pp. 5419-5422, 2002.

[2] H. Yin, A. W. Cross, W. He, A. D. R. Phelps, and K. Ronald, "Pseudospark experiments: Cherenkov interaction and electron beam post-acceleration," IEEE Trans. Plasma Sci., vol. 32, no. 1, pp. 233-239, Feb. 2004.

[3] C. S. Wong, H. J. Woo, and S. L. Yap, "A low energy pulsed X-ray source based on the pseudospark electron beam," Laser Particle Beams, vol. 25 , no. 3 , pp. 497-502, 2007.

[4] M. A. Gunderson and G. Schaefer, "Physics and applications of pseudosparks," NATO Adv. Study Inst., Ser. B, Phys., vol. 219, pp. 1-3, Jun. 1990.

[5] H. Yin, A. W. Cross, W. He, A. D. R. Phelps, K. Ronald, D. Bowes, et al., "Millimeter wave generation from a pseudospark-sourced electron beam," Phys. Plasmas, vol. 16, no. 6, pp. 063105-1-063105-6, 2009. 